Rev Biomed 2002; 13:37-41.

\title{
Toxoplasmosis gástrica en el síndrome de inmunodeficiencia adquirida.
}

\section{Caso Clínico}

Alejandro Guerrero-Flores ${ }^{1}$, Beatriz Vega-Ramos ${ }^{2}$.

${ }^{1}$ Hospital Regional Mérida ISSSTE. Departamento de Medicina Interna. ${ }^{2}$ Centro Médico Nacional "Ignacio García T". IMSS Departamento de Anatomía Patológica. Mérida, Yucatán, México.

\section{RESUMEN.}

Introducción. Toxoplasma gondii (T. gondii) es un protozoario ubicuo de aves y mamíferos, parásito intracelular obligado, cuya reactivación se observa en personas inmunodeprimidas. En el síndrome de inmunodeficiencia adquirida (SIDA), la mayoría de los casos se relacionan con infecciones del sistema nervioso central (SNC) y se ha publicado poco sobre localización gastrointestinal de esta parasitosis oportunista.

Caso clínico. Hombre de 34 años de edad atendido por fiebre irregular de 6 meses de evolución, disfagia, epigastralgia, anorexia y pérdida de peso. Una endoscopía demostró candidiasis oroesofágica y úlceras en tercio inferior del esófago, sugestivas de citomegalovirus (CMV). El paciente tenía siete años con infección VIH/SIDA sin tratamiento y presentaba anemia, anticuerpos IgG positivos contra toxoplasma y CMV. La cuenta de

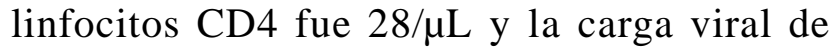

195,574 copias/mL.Recibió tratamiento con ganciclovir y fluoconazole I.V. Inició antiretrovirales combinados que suspendió por intolerancia gástrica luego de un mes. Por persistir síntomas digestivos altos, una nueva endoscopía demostró gastritis y en la biopsia se identificó $T$. gondii. El paciente falleció 2 meses después en su domicilio con síndrome de desgaste.

Discusión. Este tipo de casos se acompaña de acentuada inmunodepresión de linfocitos CD4, síntomas digestivos altos atribuibles a múltiples causas. Este es el primer caso en la Península de Yucatán de una persona con SIDA e identificación de trofozoitos de $T$. gondii en la mucosa gástrica, por lo que se debe considerar su búsqueda intencionada en pacientes con SIDA, linfocitos CD4 < 50/ $\mu \mathrm{L}$ y síntomas como anorexia, nausea, vómito, dolor abdominal en epigastrio, fiebre y diarrea.

(Rev Biomed 2002; 13:37-41) 
A Guerrero-Flores, B Vega-Ramos.

Palabras clave: $T$. gondii, toxoplasmosis gástrica, SIDA, VIH.

\section{SUMMARY.}

\section{Gastric toxoplasmosis on AIDS.}

Introduction. Toxoplasma gondii is an intracellular parasite which has been identified as an opportunistic pathogen in immunodeficienty people. During the present aids era the number of clinical cases of toxoplasmosis has incresead. The majority are associated with central nervous system infections and very few have been published regarding gastrointestinal manifestations. The few cases described report severe abdominal pain, nausea and vomiting.

Case presentation. Male, 34 years old, carrier HIV. He came to emergency room with fever of six months, anorexia, disphagia and epigastric pain. By endoscopy candidiasis was observed in the esophagus with ulcers in distal portion suggesting CMV. IgG CMV and toxoplasma antibodies were positives, anemia 8 g/dL, lymphocytes T CD4 28/ $\mu \mathrm{L}$, viral load PCR HIV amplicor $195,574 \mathrm{cop} / \mathrm{mL}$. Then received treatment for 3 weeks with ganciclovir I.V. and fluoconazole. Anti retroviral treatment was interrupted after symptomatic gastric intolerance. TMP/SMX and pyrimetamine were also administred. It was thought that the cause was gastritis medication and or reactivation of the ulcers by CMV. New endoscopy with a biopsy of the gastric mucous showed an acute gastritis secondary to the toxoplasma. Patient quit coming to appointments and died two months later at his home with wasting syndrome.

Discussion. These cases are usually accompanied by a notable immunodepression of linfocytes CD4 and strong digestive symptoms which are attributed to various causes. This is the first case that trofozoitos of $T$. gondii have been identified in the gastric mucous of an aids patients in the Yucatan
Peninsula, and therefore we should consider testing AIDS patients with linfocytes CD4 < 50/ $\mu \mathrm{L}$, and symptoms such as anorexia, nausea, vomiting, abdominal pain, fever and diarrhea for T. gondii. (Rev Biomed 2002; 13:37-41)

Key words: Gastric toxoplasmosis, AIDS, $T$. Gondii.

\section{INTRODUCCIÓN.}

Toxoplasma gondii (T. gondii), el agente etiológico de la toxoplasmosis, es un parásito intracelular obligado, que durante mucho tiempo se ha identificado como un patógeno oportunista en personas inmunodeprimidas. En los últimos 15 años se ha incrementado el número de pacientes con toxoplasmosis clínicamente sintomática, asociados al síndrome de inmunodeficiencia adquirida (SIDA) (1). En un grupo de pacientes con virus de inmunodeficiencia humana [95] y de donadores de sangre [100] en Mérida, Yucatán, México, se encontraron $47 \%$ y $69 \%$ respectivamente, portadores de anticuerpos IgG contra $T$. gondii por análisis inmunoenzimático $(2,3)$. La mayoría de los casos de toxoplasmosis en enfermos con SIDA que se han reportado se relacionan con infección del Sistema Nervioso Central (SNC), afectando el estado mental, la función neurológica, la visión, la audición y llegando hasta la muerte. En la Península de Yucatán, México, en un grupo de enfermos adultos con SIDA [260] durante un periodo de 7 años, se describió un 20\% [53] de ellos con encefalitis por $T$. gondii (4). Sin embargo este parásito puede afectar diversos tejidos y sistemas en el organismo y poco se ha publicado sobre manifestaciones gastrointestinales de esta parasitosis oportunista (5-9).

A los escasos reportes de Toxoplasmosis gástrica y a una serie de revisión de autopsias de 80 pacientes, en quienes se encontró al $6 \%$

\section{Revista Biomédica}




\section{Toxoplasmosis gástrica.}

con datos de toxoplasmosis gastrointestinal (10), añadimos un caso clínico de un paciente con SIDA, síndrome de desgaste, fiebre, dolor epigástrico de varios meses de evolución y con evidencia de gastritis y ulceraciones con trofozoitos de T. gondii.

\section{Presentación del caso clínico.}

Hombre de 34 años de edad, con serologia positiva para el virus de la inmunodeficiencia humana (VIH) en 1991, detectado como donador de sangre. Durante siete años se negó por voluntad propia a consultar por su padecimiento, presentándose hasta mayo de 1998 al servicio de urgencias del Centro Médico Nacional "Ignacio García Tellez" del Intituto Mexicano del Seguro Social en Mérida, Yucatán, México. Relatando historia de seis meses de evolución con pérdida de peso, anorexia, dolor en epigastrio, fiebre irregular y disfagia con hallazgo de candidiasis oroesofágica por endoscopía, así como úlceras en el tercio inferior del esófago sugestivas de citomegalovirus (CMV). Presentaba anemia de $8 \mathrm{~g}$ de hb, linfocitos T CD4 de $28 / \mu \mathrm{L}$, carga viral del VIH por PCR amplicor de 195, 547 copias/mL. Recibió tratamiento con fluoconazol y ganciclovir intravenoso por 21 días. Así mismo inició tratamiento antiretroviral con esquema triple basado en zidovudina, zalcitabina y saquinavir, además de trimetroprin sulfametoxazole y pirimetamina. Estos fármacos se suspendieron antes de un mes por la presencia de síntomas como dolor en el epigastrio, nausea, vómitos y disfagia baja.

Ante la sospecha de gastritis medicamentosa y/o reactivación de las úlceras del esófago se practicó una nueva endoscopía hasta el estómago encontrando datos macroscópicos de gastritis. Se realizó biopsia de la mucosa gástrica del fundus y en el estudio anatomopatológico se encontraron datos de gastritis aguda secundaria a toxoplasma (figuras 1 y 2). Se reinició tratamiento con trimetropin-

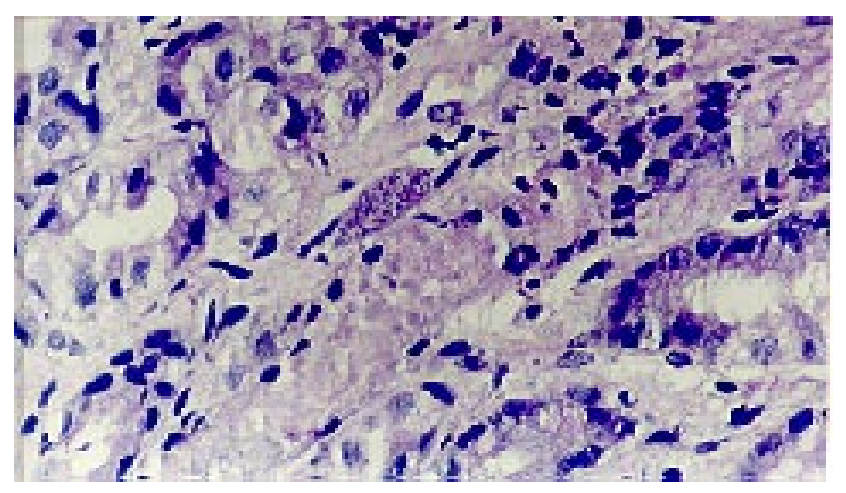

Figura 1.- Detalle microscópico en el que se identifica un quiste que contiene bradizoitos (forma latente del toxoplasma), rodeado de una leve respuesta inflamatoria de tipo agudo, (P.A.S. 400X)

sulfametoxazole. El paciente no regresó a la consulta externa y falleció dos meses después en su domicilio, con síndrome de desgaste progresivo.

\section{DISCUSIÓN.}

El presente caso clínico se suma a otros descritos con toxoplasmosis gástrica sintomática, en que puede encontrarse lesiones simultáneas por CMV (9) y un cuadro clínico similar, así como los hallazgos macroscópicos y las imágenes de histopatología.

Para T. gondii se han descrito tres formas en su ciclo de vida, trofozoitos, quistes tisulares y oocystes. Miles de trofozoitos pueden encontrarse tanto en quistes como en oocystes. Los gatos, pequeños mamíferos y los pájaros son los principales reservorios de $T$. gondii. Se conocen dos formas importantes para su transmisión, una por la ingesta de quistes tisulares u oocystes que contengan a los parásitos y por vía vertical transplacentaria madre/hijo. Después de la ingesta de quistes u oocystes los trofozoitos son expandidos en la mucosa intestinal invadiendo y multiplicándose en sus células. Después, los trofozoitos se 


\section{A Guerrero-Flores, B Vega-Ramos.}

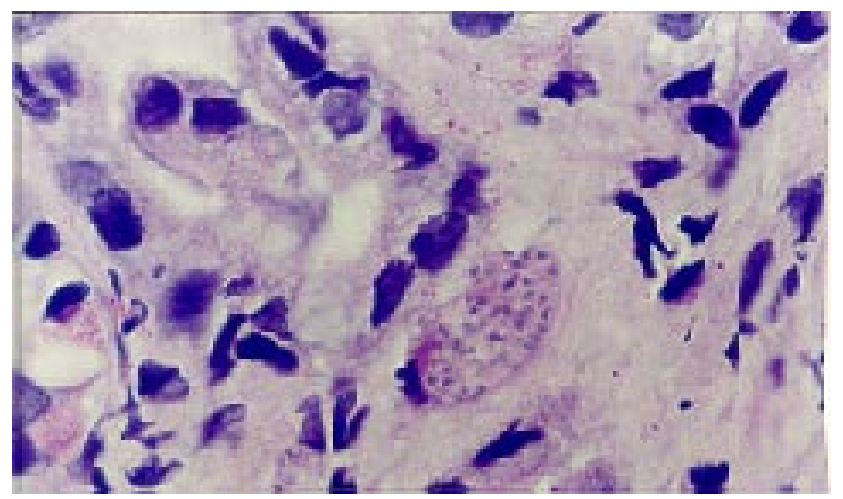

Figura 2.- Detalle histológico del quiste toxoplasmico con su pared rota, liberando taquizoitos que corresponden a la fase activa del parásito, (H.Y.E. 100X)

diseminan por la vía linfática y por la sangre infectando prácticamente todos los tejidos. Los principales órganos blanco son el músculo esquelético, miocardio, ganglios linfáticos, cerebro, retina y la placenta.

La toxoplasmosis es una parasitosis que se reactiva en personas inmunodeprimidas, con cifras de linfocitos T CD4 menores de $50 / \mu \mathrm{L}$. A pesar de esta inmunodepresión celular, hay ocasiones en que la reactivación no se presenta. Cuando ésta ocurre, afecta principalmente al SNC. Los mecanismos exactos de la respuesta inmune contra toxoplasma y las razones por las que el tubo gastrointestinal es poco afectado, no han sido establecidas completamente $(11,12)$.

Aunque esta localización es muy rara, tal como se describe en la literatura, en los pacientes con SIDA, con linfocitos T CD4 menos de $50 \mu \mathrm{L}$, síntomas digestivos altos, anorexia, disfagia, epigastralgia, nausea, vomito, fiebre y perdida de peso, es recomendable la búsqueda intencionada de $T$. gondii, con estudios microscópicos de la mucosa gastrointestinal. Este tipo de estudio permite definir el papel de esta parasitosis en el síndrome de desgaste asociado al SIDA.

\section{REFERENCIAS.}

1.- Montajer JS, LE T, Hogg R. The changing spectrum of AIDS index diseases in Canada. AIDS 1994; 8: 693-6.

2.- Góngora-Biachi RA, González-Martínez P, Castro-Sansores C, Lara-Perera D, Alonzo-Salomón G, Pavía-Ruz N, et al. Prevalencia de anticuerpos contra Toxoplasma gondii en donadores de sangre y pacientes con la infección por el virus de la inmunodeficiencia humana en Yucatán, México. Enferm infec microbiol 1996; 16:13.

3.- Góngora-Biachi RA, González-Martínez P, Castro-Sansores C, Alvarez-Moguel R, Pavía-Ruz N, Lara-Perera D, et al. Anticuerpos contra Toxoplasma gondii en pacientes con VIH en Yucatán. Rev Invest Clin 1998; 50:419-22.

4.- Guerrero A. Encefalitis toxoplasmica en el SIDA. Treinta y nueve casos clínicos. Enferm Infec Microbiol 1997; 17:65.

5.-Smart P, Weinfeld A, Thompson NE. Toxoplasmosis of the stomach: a cause of antral narrowing. Radiology 1990; 174:369-70.

6.- Florencio FRC, Albuquerque Filho FB, Moraes MAP. Toxoplasma gondii na mucosa gástrica como primeiro achado em paciente aidetico. Rev Soc Brasil Med Trop 1992; 25:275-6.

7.- Peraire J, Vidal F, Mayayo E. Gastric toxoplasmosis in the acquired immunodeficiency síndrome. Am J Gastroenterol 1993; 88:1464-5.

8.- Alpert L, Miller M, Alpert E. Gastric toxoplasmosis in the acquired immunodeficiency syndrome: antemortem diagnosis with histophatologic characterizacion. Am J Gastroenterol 1996; 110:25864.

9.- Jauntzke G, Sell M, Thalmann U. Extracerebral toxoplasmosis in AIDS: histological and immunohistological findings based on 80 autopsies. Pathol Res Pract 1993; 189:428-36.

10.- Kofman E, Khorsandi A, Sarlin J. Gastric toxoplasmosis: case report and review of the literature. Am J Gastroenterol 1996; 91:2436-38.

11.- Chardes T, Buzoni D, Lepage A. Toxoplasma gondii oral infection induces specific cytotoxic cd8

\section{Revista Biomédica}




\section{Toxoplasmosis gástrica.}

a thy 1 gut intraepithelial lymphocytes, lytic for parasite infected enterocytes. J Immunol 1994; 50:4596- 603 .

12.- Chardes T, Bout D. Mucosal immune response in toxoplasmosis. Res Immunol 1993; 144:57-60. 